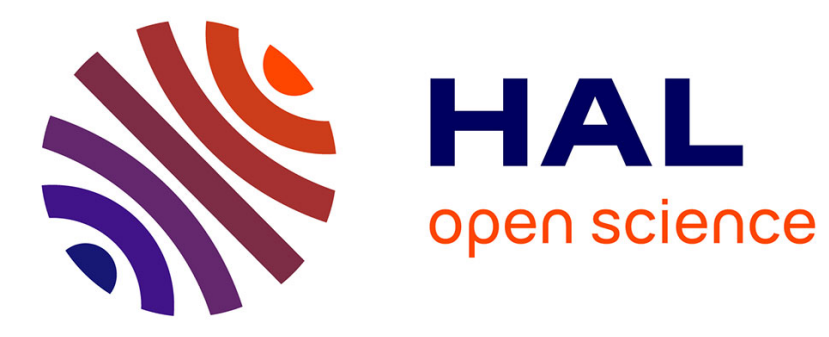

\title{
Smart distance keeping: modeling and perspectives for embedded diagnosis
}

\author{
Hassan Shraïm, Othman Nasri, Philippe Dague, Olivier Héron, Mickael
}

Cartron

\section{- To cite this version:}

Hassan Shraïm, Othman Nasri, Philippe Dague, Olivier Héron, Mickael Cartron. Smart distance keeping: modeling and perspectives for embedded diagnosis. 1rst International Conference on Intelligent Systems, Modelling and Simulation ISMS'10, Jan 2010, Liverpool, United Kingdom. inria-00540819

\section{HAL Id: inria-00540819 \\ https://hal.inria.fr/inria-00540819}

Submitted on 29 Nov 2010

HAL is a multi-disciplinary open access archive for the deposit and dissemination of scientific research documents, whether they are published or not. The documents may come from teaching and research institutions in France or abroad, or from public or private research centers.
L'archive ouverte pluridisciplinaire HAL, est destinée au dépôt et à la diffusion de documents scientifiques de niveau recherche, publiés ou non, émanant des établissements d'enseignement et de recherche français ou étrangers, des laboratoires publics ou privés. 


\section{SMART DISTANCE KEEPING: MODELING AND PERSPECTIVES FOR EMBEDDED DIAGNOSIS}

\author{
Hassan Shraim \\ LRI, University of Paris-sud 11, CNRS \\ \& INRIA Saclay - Ile-de-France \\ Bât 490, 91405 Orsay Cedex France \\ Email: hassan.shraim@gmail.com \\ Olivier Heron \\ CEA LIST, Saclay \\ 91191 Gif-sur-Yvette cedex France \\ Email: olivier.heron@cea.fr
}

\author{
Othman Nasri \\ LRI, University of Paris-sud 11, CNRS \\ \& INRIA Saclay - Ile-de-France \\ Bât 490, 91405 Orsay Cedex France \\ Email: othman.nasri@lri.fr
}

\author{
Philippe Dague \\ LRI, University of Paris-sud 11, CNRS \\ \& INRIA Saclay - Ile-de-France \\ Bât 490, 91405 Orsay Cedex France \\ Email: philippe.dague@lri.fr
}

\author{
Mickael Cartron \\ CEA LIST, Saclay \\ 91191 Gif-sur-Yvette cedex France \\ Email: mickael.cartron@cea.fr
}

\begin{abstract}
This paper presents a detailed description of an advanced Adaptive Cruise Control (ACC) system implemented on a Renault-Volvo Trucks vehicle. One of the main differences between this new system, which is called the Smart Distance Keeping (SDK), and the classical ACC is the choice of the safe distance. This later is the distance between the vehicle (with the ACC or the SDK system) and the front obstacle (which may be a vehicle). It is supposed fix in the case of the ACC, while variable in the case of the SDK. The variation of this distance (in the case of SDK) depends essentially on the relative velocity between the vehicle and the front obstacle. The choice of this distance influences the velocity regulation. The main contribution of this work is on the SDK system architecture, the design of its environment model, and the proposition of a detection and isolation strategy for some of the possible faults that may be produced on the system.
\end{abstract}

Keywords-Smart Distance Keeping (SDK), vehicle modeling, fault diagnosis.

\section{INTRODUCTION}

An increasing number of vehicles are being equipped with adaptive cruise control (ACC). This technology is substantially more complex than conventional cruise control; ACC adjusts the brake and/or throttle, within limited ranges, to maintain a constant headway from any vehicle that intrudes upon the path of the driver's vehicle. While ACC provides a potential safety benefit in helping drivers to maintain a constant speed and headway [1], as with other types of automation, there is the potential for misuse and disuse [2]. Adaptive cruise control (ACC) provides assistance to the driver in the task of longitudinal control of his vehicle during motorway driving. The system controls the accelerator, engine power train and vehicle brakes to maintain a desired time-gap to the vehicle ahead.

For ACC to be effective, drivers need to understand the capabilities of the technology, which include braking and sensor limitations. Based on this understanding, they must be able to intervene when a given situation exceeds ACC capabilities. However, drivers have difficulties in understanding how ACC functions [3]. As a result, they tend to rely on the system inappropriately. For instance, [4] showed that drivers failed to intervene when approaching a stopped queue of vehicles because they believed that the ACC could effectively respond to the situation. [6] introduced an unexpected acceleration into the ACC system during routine driving conditions, which resulted in a collision 33 percent of the time. Whether or not drivers can respond effectively when automation fails depends on their understanding of the type of failure that occurs and the context in which it occurs [5].

To ensure safe and effective use, ACC limits of operation should be identifiable and interpretable [7]. One approach to help drivers detect and respond to these limits is to match the limits of the ACC algorithm to the natural boundaries drivers use to switch between car-following and active braking behaviors, as defined by environmental cues [e.g. time headway (THW) and time to collision (TTC)].

Essentially, this means matching the function of the ACC to the way drivers perform the task of following other vehicles [7]. Individual differences in driving behavior, however, would require some degree of tuning of the ACC algorithm to individual drivers.

The SDK must be understood as a function to enhance the driver's capability to manage his longitudinal environment, and is dedicated to a use on highways or expressways (straight line, low curvatures, one-way roads). The SDK is based on the immediate front environment sensing on one hand, and on the automated management of the truck longitudinal actuators (brakes, engine, gearbox), all this being monitored and controllable at any time by the driver through the in-cabin human machine interface and the conventional driving commands (pedals, switches). 
Sensor outputs should always be checked to ensure that they are within their expected operating range. Simple checks on the recent rate of change or variance of the output can also be incorporated. Faults which cause the sensor to have an offset or altered gain will affect the control system but may not be detected by this first level approach.

The traditional approach to sensor fault checking is to include hardware redundancy for sensors. If two sensors measuring the same quantity disagree, there is likely to be a fault in one of them, and if three or more measurements are available, the fault is likely to be in the sensor which disagrees most.

The high cost of providing direct hardware redundancy for sensors has led to the development of analytic redundancy techniques. Conceptually, this equates to creating virtual sensors from other available measurements, to compare with the one being monitored. Analytic redundancy is used in available passenger car control systems.

The paper is structured as: first we begin by a general description and modeling of the SDK controller with its environment system. The modeling in this work includes a simplified mathematical model of the wheels and the engine. Then, a strategy for the detection and the isolation of the some of the possible faults that may be produced on the overall system is shown. Finally test scenarios and conclusion are presented.

\section{SYSTEM DESCRIPTION AND MODELING}

\section{A. SDK System}

In our work, we are interested on the modeling and the diagnosis of the SDK system. On the diagnosis part, we concentrate on the sensors fault diagnosis (truck velocity sensor, wheels angular velocity sensors, radar, engine sensors). We suppose during this study that no faults may be produced on the bus CAN or on coding the algorithms (software).

The main architecture of the global system can be represented by figure 1 . As shown in this figure, the global SDK system may be decomposed into two main parts, the SDK controller, and the SDK environment model. The main function of the SDK controller is to:

- find the minimal distance between the truck and the front object,

- find the acceleration (deceleration) needed to realize the correct functioning of the SDK system,

- use a control algorithm to control the engine.

Inputs to the controller:

- relative velocity between the SDK vehicle and the front vehicle,

- distance between the SDK vehicle and the front vehicle,

- type of the front object (moving or not moving object),

- velocity of the SDK vehicle which may be found by one of the following ways:

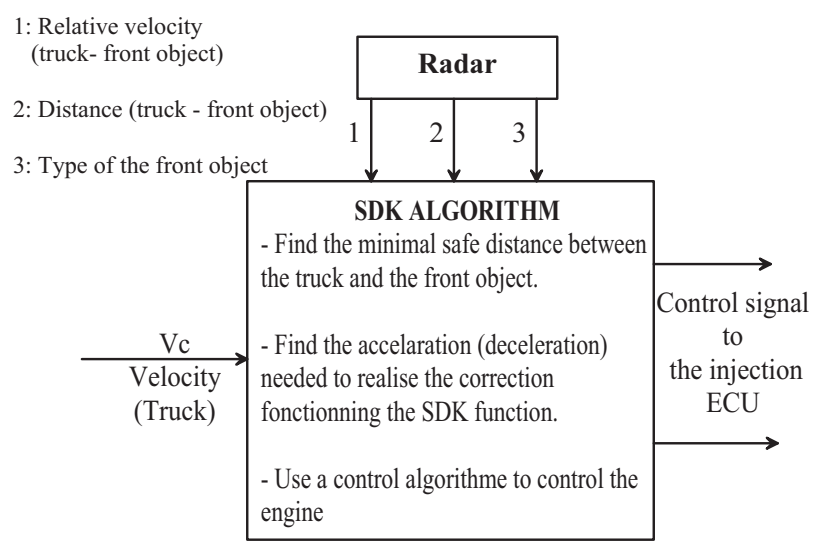

Figure 1. SDK system: algorithm and environment

- a velocity sensor (Tachograph, or from the transmission system),

- the average value of wheels angular velocity measurements.

Outputs from the controller:

- control of the injection system,

- information sent to the Human Machine Interface about the status of the SDK system.

By showing this architecture, we see that the decision of the SDK controller depends essentially on the data issued from some sensors, which means, any faulty data, will influence the SDK system decision.

In order to avoid as maximum as possible the faulty data propagation, we analyze in this work the following element: the radar, the wheel, the engine, and the communication buses.

1) The Radar: The SDK needs to be informed about the front object presence, and about its relative position and velocity. Within this work, the sensor is a 3 beam Doppler effect ARS100 Radar. This radar monitors the traffic in front of the vehicle using three stationary independent millimetre wave radar.

Moving and stationary objects are detected and their distance and relative velocity are measured and processed sixteen times per second.

Due to its physical nature, the radar sensor is offering excellent performance characteristics even in adverse weather conditions.

Since the data issued from the radar depends on the external object, then in order to realize any simulation, several scenarios should be prepared for the movement SDK vehicle and the front object. In this work, we suppose that the distance and the relative velocity between the SDK vehicle and the front vehicle are inputs to the system (depending on the scenarios that we are choosing). 
2) The Wheels: Wheel angular velocity is one of the important inputs to the SDK controller. Based on the angular velocities (six wheels), the linear truck velocity is calculated.

In this part, the wheel rotational dynamics are presented. By applying Newton's Law to the dynamics of the wheels, we find:

$$
I_{w_{i}} \dot{w}_{i}=R_{i} F_{X_{i}}+\operatorname{Torque}\left(m_{i}\right)-\operatorname{Torque}\left(b_{i}\right)
$$

Where $I_{w_{i}}$ is the moment of inertia of the wheel number $i, R_{i}$ is the effective radius, $w_{i}$ is the angular velocity of the wheel, Torque $\left(m_{i}\right)$ is the applied tractive torque, and Torque $\left(b_{i}\right)$ is the braking torque.

\section{B. Motor and Power Train}

Modern diesel engines are essentially decomposed into several subsystems [10]: the exhaust manifold, the intake manifold, the injection (common rail), the engine with moving solids (rods, pistons, cylinders ...), the turbo charge. Each of these subsystems can be modeled separately, but for some of these subsystems it is difficult to develop analytical models, so static maps based on the knowledge about the system may be used.

The presented problem in this section is to develop a simplified model for the diesel engine (see figure 2) used by the SDK. Based on [10], the dynamics of motor rotation is given by (2).

$$
J_{e} \dot{w}_{e}(t)=M_{\text {ind }}\left(t-\tau_{i}\right)-M_{f}(t)-M_{\text {load }}(t)
$$

Where $w_{e}$ is the crank shaft angular velocity, $M_{i n d}$ is the indicated torque, $\tau_{i}$ is the delay, $M_{f}(t)$ is the friction torque, $M_{\text {load }}$ is the torque due to the load, and $J_{e}$ is the effective inertia of the engine.

In this work, the transmission system is represented as in the figure 3. For simplification purposes, the "Transmission System" block is composed only of several constants, depending on the gearbox state.

1) The Admission and Intake Manifold: The temperature $T_{i m}$ of the intake manifold is assumed to remain constant due to the intercooler. Therefore, the analysis will be based on essentially on the variation of the pressure. In this study, the input flow is characterized by the output flow $P_{i m}$ of the compressor. In this study, the input flow is characterized by the output flow $\dot{m}_{c}$ of the compressor (see equation (3)).

$$
\dot{P}_{i m}+\frac{\tau_{v} V_{d} N_{e}}{120 V_{i m}} P_{i m}=\dot{m}_{c} \frac{R T_{i m}}{V_{m}}
$$

Where $V_{i m}$ is the volume of the intake manifold, $\tau_{v}$ is the volumic efficiency, $V_{d}$ is the exchange volume in the engine, $\dot{m}_{c}$ is the rotational velocity of the engine in $\operatorname{rpm}\left(N_{e}=\right.$ $\left.\frac{60 w_{e}}{2 \pi}\right)$.

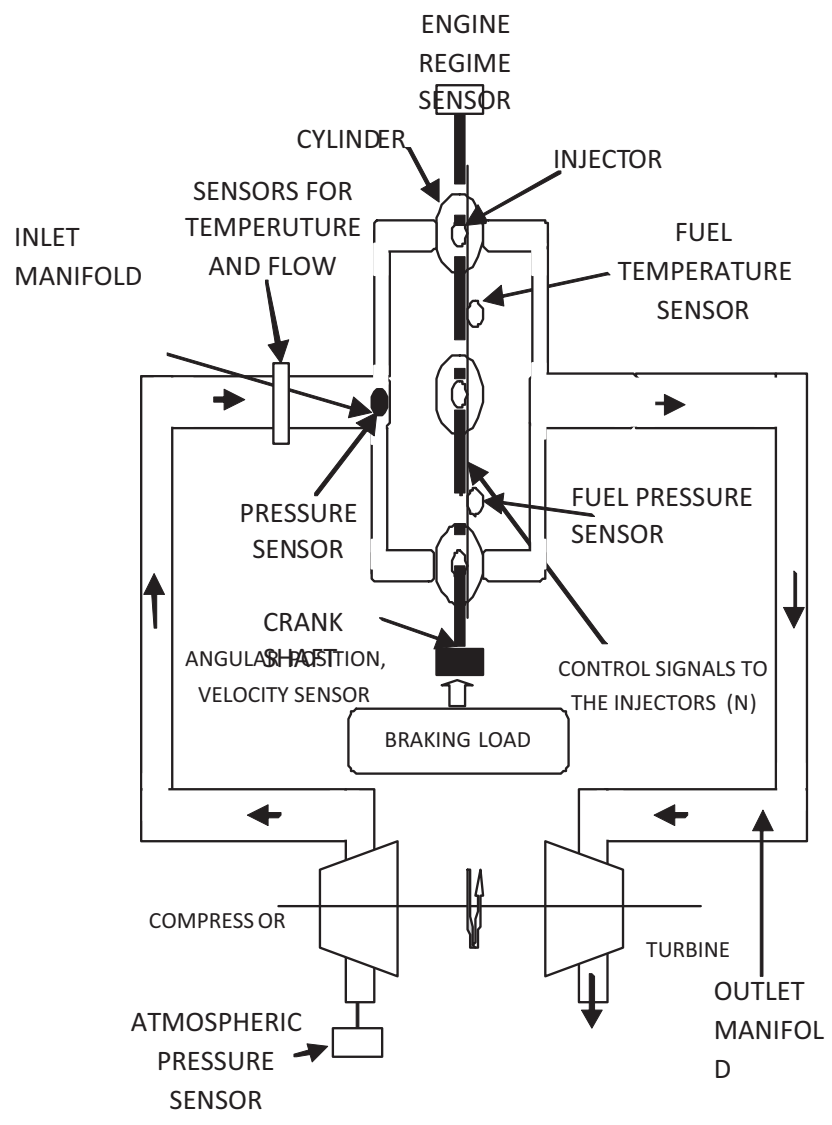

Figure 2. Engine Architecture, [10]

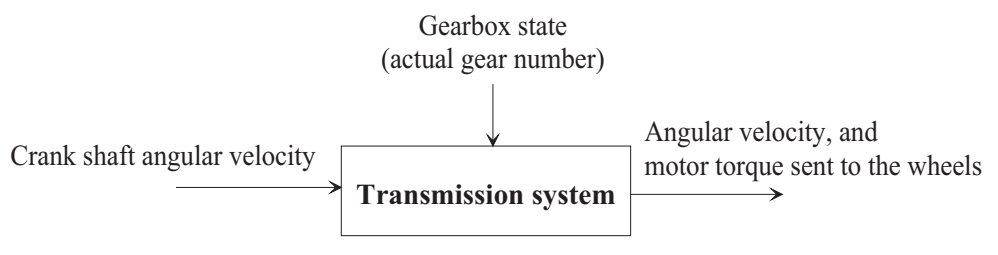

Figure 3. Transmission block

2) The Indicated Torque $M_{\text {ind }}$ : In order to calculate the indicated torque, we should calculate firstly the indicated efficiency. Normally, this efficiency is a specific characteristic to the engine and it is found from empirical data. This efficiency is higher when the mixture is light, and it may be approximated by (4).

$$
\mu_{i n d}=a+b \lambda+c \lambda^{2}
$$

The coefficients $(a, b, c)$ are found by identification (three different tests have been made) and $\lambda$ is defined by (5).

$$
\lambda=\frac{\frac{P_{i m} w_{e} V_{d}}{4 \pi R} \tau_{v}}{T_{i m} \dot{m}_{f}}
$$


Then the indicated torque $M_{\text {ind }}$ can be found as follows:

$$
M_{i n d}=m_{f} p_{c_{i}} \mu_{i n d}
$$

$p_{c_{i}}$ is a characteristic for the diesel $\left(40000000 \mathrm{~J} \mathrm{Kg}^{-1}\right)$.

3) The Injection: The injection system controls the quantity of fuel that will be introduced into the combustion chamber. The mixture Air/fuel should be capable to auto ignited by the effect of temperature and the high pressure. The calorific power of combustion is related to the quantity of fuel injected. The following model gives the flow of fuel $m_{f}$ in function of the accelerator pedal $x_{p}$ and the engine speed (see equations (7) and (8)) [10].

$$
\begin{aligned}
\dot{m}_{f} & =i_{0}+\Delta \dot{m}_{(S D K)}+\Delta \dot{m}_{f} \\
\Delta \dot{m}_{f} & =w_{e}\left(i_{1}+i-2 x_{p}+i_{3} x_{p}^{2}+i_{4} w_{e}\right)
\end{aligned}
$$

Where $i_{0}$ characterizes the minimal injection flow (greater than zero, when the engine is idle), $m_{f}$ models the variations of the flow around, $i_{0}, i_{1}, \ldots, i_{4}$ are constants.

4) The Friction Torque $M_{f}$ : The friction torque may be calculated by (9).

$$
M_{f}=\frac{\left(c_{0}+c_{1} w_{e}+c_{1} w_{e}^{2}\right) V_{d}}{2 \pi n r}
$$

5) The Load Torque $M_{\text {load: }}$ The torque $M_{\text {load }}$ depends on the type of the road, the vehicle velocity, the turnings,... In this work we will suppose that this torque is input to the system and it has a constant value.

\section{POSSIBLE FAULTS AND DIAGNOSIS STRATEGY}

In this section, we present the possible faults that may be produced and diagnosis strategy realized.

\section{A. Radar Data}

1) Detection Performance: The basic data detection requirement is to measure distance, relative speed, and reflection signal amplitude of moving and stationary objects in three beams. Angular position is calculated by the interpolation algorithms based on signal levels in adjacent beams.

2) Several Scenarios For the Radar Fault Detection Analysis:

i. First: if the radar is faulty and doesn't detect any object. So, without the help of another device, we can do nothing.

ii. Second: if the radar works but gives incorrect distances (with a certain shift of $x$ meters): for example $(150 m \rightarrow 150-x m)$ where $x$ is a constant term, then, we cannot detect this fault.

iii. Third: if the relative velocity and the distance between the vehicles are measured separately (two different measurement tools), then it is important to check at each period (for example 2 seconds) if the variation in the distance corresponds to the variation in the relative velocity. If there is a difference then we say that there is a fault.

Ex: suppose that we initially have the relative velocity $V_{\text {rel }}$ and the distance $d$ between the SDK truck and the front vehicle $A V$ see figure 4, so if we consider that the period (that we choose for checking) is equal to 2 seconds, then we should obtain

$$
d(t)-d(t-2)=2 * \operatorname{Avera}\left(V_{\text {rel }}\right)
$$

Where $\operatorname{Avera}\left(V_{\text {rel }}\right)$ is the average value of $V_{\text {rel }}$ in the period of 2 seconds.

iv. Fourth: Suppose that the radar was detecting a front $A V$; vehicle (see figure 4).

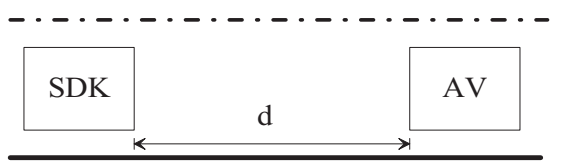

Figure 4. SDK Vehicle and the front vehicle AV

Let $V_{\text {rel }}$ be the relative velocity between the two vehicles then: $V_{r e l}=V_{a v}-V$. As we have shown before, the relative velocity is a measurement given by the radar. And also, the SDK vehicle velocity $V$ is measured from other sensors (wheels angular velocities or vehicle velocity), then we can find the velocity of the $A V$ vehicle.

Getting the velocity of the front vehicle we can analyse as follows:

If there is a strong sudden variation (and then its acceleration (deceleration) is not realistic) then we have one of the two following cases:

i. there is a fault in the radar sensor,

ii. the value of the SDK vehicle velocity is faulty,

iii. an intruder vehicle comes in front of the SDK vehicle (see figure 5).

In all the above cases, it is important to observe the velocity of the AV vehicle for several points before taking any decision.

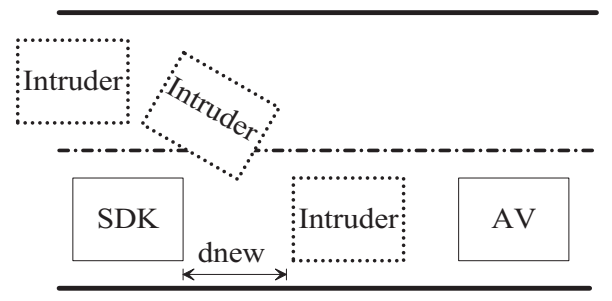

Figure 5. SDK Vehicle and the front vehicle AV with intruders (vehicles)

In some of the above cases, the calculation of the AV vehicle acceleration (deceleration), may give a non realistic 
values. A study about the maximum (minimum) possible acceleration (deceleration) can be given as follows:

$$
\begin{aligned}
\left|a_{x}\right|_{\max } & =\max \left|\frac{\sum F_{x_{i j}}}{m}\right|=\max \left|\frac{\mu \cdot N_{z}}{m}\right|=\max \left|\frac{\mu \cdot m . g}{m}\right| \\
& \leq \mu_{\max } . g
\end{aligned}
$$

A maximum friction coefficient $\left(\mu_{\max }\right)$ determines maximum acceleration or deceleration. It can be estimated using sliding modes techniques as follows (see the following section). Then if a non realistic acceleration (deceleration) value is found, then if there is no intruder vehicle, we can suppose that there is radar fault.

The overall diagnosis block for the Radar can be represented by the figure 6 .

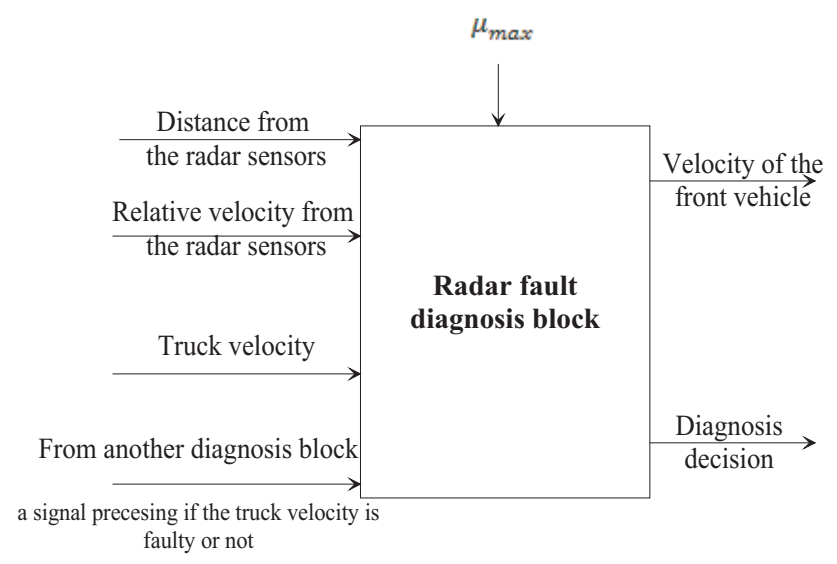

Figure 6. Radar fault diagnosis block

\section{B. $\mu_{\max }$ Estimation}

In order to estimate $\mu_{\max }$, sliding modes observers can be applied. A hierarchical observer is needed for this estimation. In the first step, an observer based on the dynamical equation of the wheels should be developed. This observer takes as an input the applied motor torque (which is estimated statically (existing maps)) and the braking torque, which can be easily found based on the hydraulic pressure sent to the wheels [8]. Then, in parallel to this observer, a sliding modes observer is used to estimate the vertical forces. This observer is based on the suspension system modeling [9]. Then by calculating the longitudinal force and the vertical force we apply the following formula to estimate the adherence coefficient:

$$
\mu_{\max }>\frac{\max \left(\sum_{i=1}^{n} F_{x_{i}}\right)}{\min \left(\sum_{i=1}^{n} F_{z_{i}}\right)}
$$

Where $n$ is the number of the wheels (equals to 6), $F_{x_{i}}$ and $F_{z_{i}}$ are respectively the longitudinal and the vertical forces applied at the wheel $i$.
Table I

ANGULAR VELOCITY COMPARISON FOR THE WHEELS $1,3,5$

\begin{tabular}{|l|c|c|c|}
\hline Difference & $w_{1}-w_{3}$ & $w_{1}-w_{5}$ & $w_{3}-w_{5}$ \\
\hline$f_{\text {odd } i}$ & $f_{13}$ & $f_{15}$ & $f_{35}$ \\
\hline
\end{tabular}

Table II

ANGULAR VELOCITY COMPARISON FOR THE WHEELS 2, 4, 6

\begin{tabular}{|l|c|c|c|}
\hline Difference & $w_{2}-w_{4}$ & $w_{2}-w_{6}$ & $w_{4}-w_{6}$ \\
\hline$f_{\text {even } i}$ & $f_{24}$ & $f_{26}$ & $f_{46}$ \\
\hline
\end{tabular}

Table III

COMPARISON OF THE ANGULAR VELOCITY OF THE WHEELS: 1, AND 2, 4,6

\begin{tabular}{|l|c|c|c|}
\hline Difference & $w_{1}-w_{2}$ & $w_{1}-w_{4}$ & $w_{1}-w_{6}$ \\
\hline$f_{1 i}$ & $f_{12}$ & $f_{14}$ & $f_{16}$ \\
\hline
\end{tabular}

\section{Truck Velocity}

The truck velocity used in the embedded SDK system is found by calculating the mean value of the wheels angular velocities. The truck velocity is an important decision term for the SDK controller. So, in order to ensure that the truck velocity value is not faulty, we analyze the rotational velocity value of each wheel. A simple fault detection strategy (based on the comparison between wheels velocities) aiming to detect wheels angular velocities sensors fault is proposed. To realise this study, two important steps are done:

1) A Simulator for the Truck Model: A Matlab Simulink simulator representing the truck model is prepared. All the equations presented previously in the paper are coded in this simulator. The angular velocities of the wheels are found by equations 1 , where the motor torque is found based on the angular velocity of the crank shaft (by a static map). The braking torque also is an input to these equations. This torque can be calculated based on the hydraulic brake pressure sent to the wheel [8].

2) A Detection Strategy: Two cases are considered based on steering angle:

i. Case of straight line motion: In this case, we suppose that the angular velocities of the six wheels should be approximately equal. Then in order to apply this strategy, we suppose that we have two groups: group 1 (for the wheels: $1,3,5$ ), and group 2 (for the wheels: $2,4,6)$, and we calculate the differences in the angular velocities as shown in table I and table II. Then if $\left(w_{i}-w_{j}\right)<\epsilon$, then we suppose that there is no fault, and $f_{i j}=0$, else we have a fault and $f_{i j}=1$. To localise the fault in the case of $f_{i j}=1$, a small algorithm is realized. This algorithm is able to localise from 1 to 4 faults. In the case of more than four, it gives a signal that all the wheels are faulty.

The realization of this algorithm is based on the tables 1, 2, 3, 4, 5. By completing these tables, the localisation of the fault will be evident. 
Table IV

COMPARISON OF THE ANGULAR VELOCITY OF THE WHEELS: 3 , AND 2 ,

4,6

\begin{tabular}{|l|c|c|c|}
\hline Difference & $w_{3}-w_{2}$ & $w_{3}-w_{4}$ & $w_{3}-w_{6}$ \\
\hline$f_{3 i}$ & $f_{32}$ & $f_{34}$ & $f_{36}$ \\
\hline
\end{tabular}

Table V

COMPARISON OF THE ANGULAR VELOCITY OF THE WHEELS: 5, AND 2 , 4,6

\begin{tabular}{|l|c|c|c|}
\hline Difference & $w_{5}-w_{2}$ & $w_{5}-w_{4}$ & $w_{5}-w_{6}$ \\
\hline$f_{5 i}$ & $f_{52}$ & $f_{54}$ & $f_{56}$ \\
\hline
\end{tabular}

ii. Case of a curve motion: In this case, we follow the same strategy proposed in the previous case, with the five tables, but the main difference here, in the case of the curve, is when we compare a wheel in group 1 (for the wheels : 1, 3, 5) to a wheel in group 2 (for the wheels: $2,4,6$, we should take in consideration a small difference that can be calculated geometrically based on the Ackerman angle theory and based essentially on the steering angle. And then we should replace $\epsilon$ by $\epsilon^{\prime}$.

In addition to the decision about faulty sensors, the truck real velocity can be also calculated in this algorithm, this velocity is the actual real velocity which is approximated based on the non faulty sensors in the case that the number of the faulty sensors is less than four.

\section{TEST CONDITIONS AND SIMULATION ENVIRONMENT}

First, mathematical models are validated by the company Renault-Volvo Trucks (Lyon Section in France). Several tests have been realized and the results were reasonable. Then, the above functions and algorithms are coded in the $\mathrm{C}$ language and implemented in the microcontroller card (prepared by the company Serma Engineering). The supervisor's role is to treat all CAN messages, sent to it in CANoe. From these messages, it retrieves information required for diagnosis, applies the algorithm developed by the global ECU and sends to other ECUs through Can-bus network the diagnostic results and the counter-reactions. In addition, in order to visualize the diagnosis results and the supervisor activity, a PC interface is used by RS232 link. It allows communication between the supervisor and a HMI that displays progressively the information provided by the supervisor.

\section{CONCLUSIONS}

In this work, we have presented the architecture of the SDK system, with simplified models for the Engine and the wheels. A simplified strategy for the faults detection and isolation is also presented. Based on the analysis that we have done, we conclude that the proposed strategy for the detection and the isolation of faults (especially wheels angular velocities) may improve the performance of the SDK system and increase its safety level.

\section{REFERENCES}

[1] Davis, L.C. 'Effect of Adaptive Cruise Control Systems on Traffic Flow'. Physical Review E, 69(6), 2004.

[2] Parasuraman R. Riley V. 'Humans and Automation: Use, Misuse, Disuse, Abuse', Human Factors, 39(2), pp. 230-253, 1997.

[3] Stanton, N.A., Marsden, P. (1996). From fly-by-wire to driveby-wire: Safety implications of automation in vehicles. Safety Science, 24(1), pp. 35-49, 1996.

[4] Nilsson, L. Safety effects of adaptive cruise control in critical traffic situations, Proceedings of the Second World Congress on Intelligent Transport Systems: Steps Forward, Vol. III, (Yokohama: VERTIS), pp. 1254-1259, 1995.

[5] Lee, J. D., See, K. A. 'Trust in technology: Designing for appropriate reliance'. Human Factors, 46(1), pp. 50-80, 2004.

[6] Stanton, N.A., Young, M., McCaulder, B. 'Drive-by-wire: the case of driver workload and reclaiming control with adaptive cruise control'. Safety Science 27 (2), pp. 149159, 1997.

[7] Goodrich, M.A., Boer, E.R., 2003. 'Model-based humancentered task automation: a case study in ACC system design'. IEEE Transactions on Systems, Man, and Cybernetics Part ASystems and Humans 33 (3),pp. 325336, 2003.

[8] Shraim H., , "Modeling, Estimation and control for vehicle dynamics", PhD Thesis, University Paul Czanne Aix Marseille III, 2007.

[9] Imine H. and Dolcemascolo V., "Sliding Mode observers to heavy vehicle vertical forces estimation ", IJHVS, International Journal of Heavy Vehicle Systems, 2008, Vol. 15, No.1 pp. 53-64.

[10] Peysson F. , Noura H., Younes R., 'Diagnostic de défauts sur un moteur diesel', CIFA06, 2006, Bordeaux, France. 